\title{
Llanos, E. (2016). La democracia y el progreso providencial. Análisis e interpretación de los conceptos: democracia, república, progreso y providencia en la filosofía política desarrollada en la Facultad de Letras de San Marcos a fines del siglo XIX y principios del XX (1869-1909). Lima, Perú: Fondo Editorial de la Universidad Nacional Agraria La Molina.
}

La democracia y el progreso providencial es un libro que analiza e interpreta conceptos como democracia, república, progreso y providencia en la filosofía política desarrollada en la Facultad de Letras de la Universidad Nacional Mayor de San Marcos (UNMSM), entre 1869 y 1909. Se centra en la revisión de una muestra de tesis de grado que se elaboraron en un periodo de transformaciones, en el marco de las reformas universitarias de 1866 . Dentro de ellas se encuentra la creación de dicha Facultad y, por tanto, los inicios de la producción académica filosófica en esa casa de estudios sobre los principales sucesos de la realidad nacional.

El primer capítulo contiene una introducción con los principales antecedentes y características del contexto, así como las corrientes filosóficas que influyeron en el desarrollo de estas tesis, entre las cuales destacan el liberalismo, la ilustración, el positivismo y el socialismo. Posteriormente, en el segundo capítulo, se explica el concepto de democracia y su diferencia con el de república, y cómo el significado de estos ha evolucionado y se ve reflejado en los documentos analizados. En el tercer capítulo, se precisa la definición de providencia y progreso, así como los cambios que han surgido desde sus inicios hasta la etapa de trabajo en la que se circunscriben los estudios sanmarquinos. Por último, el capítulo cuarto concluye con un análisis relacional de los conceptos de providencia y progreso, concatenados también con los de democracia y república.

El libro de Ernesto Llanos tiene significativos aportes. Uno de ellos es recordar que en la elaboración de investigaciones es clave valorar la perspectiva histórica 
de lo que se quiere conocer o explorar. Hurgar en el pasado no solo es una acción que ejercita la memoria individual y colectiva sobre un hecho determinado, sino que además permite viajar en el tiempo para establecer asociaciones e interpretaciones sobre eventos relevantes que ocurren en el presente y que pueden dar pistas para analizar las posibles tendencias en el futuro. Es por ello que el autor ofrece un recorrido histórico sobre cómo se desarrolló el pensamiento filosófico en los asuntos políticos-sociales a fines del siglo XIX y principios del siglo XX, develando la importancia de conocerlo hoy en día.

Por otro lado, nos invita a pensar sobre cómo los intereses de estudio en las universidades públicas son influenciados por diferentes corrientes y acontecimientos ocurridos en una época específica y, en consecuencia, cómo está correlacionado con el planteamiento de conclusiones sobre la problemática estudiada. Ello cobra especial importancia en un escenario de reciente aprobación de la Ley Universitaria n. ${ }^{\circ}$ 30220, que al igual que en 1866 impulsa una serie de cambios, y las implicancias que podría tener en la producción de investigaciones.

Así también, la contribución del estudio trasciende el ámbito filosófico pues aborda temas neurálgicos como la democracia —entendida como una forma de gobierno en la cual el pueblo tiene el poder de decidir-y la república — definida como un sistema político fundado en las leyes y en la igualdad-. Estos requieren ser repensados, especialmente en sociedades como la nuestra cuya trayectoria ha tenido diversos eventos y procesos que han vulnerado la democracia, lo cual ha ocasionado efectos perjudiciales para la atención de las necesidades ciudadanas, las brechas de desigualdad y el relacionamiento entre el Estado y la ciudadanía.

Finalmente, recomiendo este texto ya que también logra el propósito de generar en el público lector nuevas interrogantes intelectuales e interpelaciones 
ciudadanas sobre la democracia y la providencia, esta última asociada con la creencia del poder supremo de Dios y su intervención en la historia de la humanidad. Particularmente, el texto me genera las siguientes preguntas: en la actualidad, ¿cómo se manifiestan las prácticas democráticas y providenciales en los espacios públicos de poder?; ¿qué factores contribuyen a facilitar o dificultar la presencia de estas?, ¿cómo influyen en la toma de decisiones sobre los asuntos de interés público?; ¿contribuyen las prácticas providenciales a promover una mejor convivencia en igualdad y el ejercicio de derechos?; ¿cuáles son los desafíos de las universidades públicas y de la filosofía en ese sentido?; ¿cómo asumimos nuestra responsabilidad en el fortalecimiento de la democracia?

Rosario De La Cruz Huamán, Universidad Nacional Mayor de San Marcos delacruzcharo@yahoo.es 